\title{
Verletzungen der unteren Extremität im Kindesalter
}

\author{
Dorien Schneidmüller, Emanuel V. Geiger, Johannes Frank, Ingo Marzi
}

\section{Zusammenfassung}

Die Therapie der Verletzungen der unteren Extremität hat sich in den letzten Jahren zunehmend von der konservativen Therapie zur operativen Therapie und damit meist frühfunktionellen Nachbehandlung gewandelt. Je nach Frakturlokalisation sind Spontankorrekturen von Fehlstellungen zwar möglich, können aber zu signifikanten Beinlängendifferenzen führen. Daneben steigen die Ansprüche an die Mobilität aus sportlichen und sozialen Gründen. So hat sich in der operativen Behandlung der Schaftfrakturen die intramedulläre elastische Nagelung mit Titannägeln durchgesetzt. Metaphysäre Frakturen und Wachstumsfugenlösungen können meist geschlossen reponiert und mittels Drähten, aber auch Schrauben, Fixateur externe oder intramedullärer Nagelung stabilisiert werden. Epipyhsenfrakturen müssen als Gelenkfrakturen wie beim Erwachsenen auch bei Dislokation offen reponiert und mittels Kompressionsosteosynthese fixiert werden. Neben der Gefahr der inkorrekten Technik besteht bei noch offenen Wachstumsfugen die Gefahr der Entwicklung von Wachstumsstörungen, welche stets mitberücksichtigt werden müssen. Aus diesem Grunde erfolgt auch bei regelrechter Frakturheilung und Verlauf eine klinische Nachkontrolle bis zu 2 Jahre nach dem Unfall bzw. bis zum Wachstumsabschluss. Auch in der Behandlung von Kniebinnenverletzungen, wie der intraligamentären Kreuzbandruptur, und der Patellaluxation hat sich das Therapiekonzept in den letzten Jahren grundlegend verändert. Um Instabilitäten und Spätschäden zu vermeiden, erfolgt bereits im Kindesalter ein operativer Ersatz des vorderen Kreuzbands auch bei offenen Wachstumsfugen. Bei der Patellaluxation hat sich bei der primären, aber auch sekundären medialen Stabilisierung als weiteres Verfahren die Naht und Rekonstruktion des MPFL (mediales femoro-patellares Ligament) etabliert.

\section{Lower Limb Injuries in Children}

The therapy for lower limb injuries has changed in the past few years from mainly conservative therapy to an increasing degree of surgical treatment and thus to early functional rehabilitation. Depending on the localisation of the fracture spontaneous corrections or misalignments are possible but can lead to significant differences in leg length. At the same time the demand for mobility has increased due to sports and social reasons. Thus intramedullary elastic nailing with titanium pins has become established in the surgical treatment of shaft fractures. Metaphyseal fractures and growth plate detachments can mostly be repositioned in a closed technique and stabilised by wires, or also screws, external fixateurs or intramedullary nailing. As in adults epiphyseal fractures, also with dislocation, must be treated as joint fractures, repositioned in an open technique, and stabilised with osteosynthesis compression plates. Besides the danger of using an inappropriate technique, in the case of open growth plates there is a risk for the development of growth disorders and this must always be taken into consideration. For these reasons, after completed fracture healing and course a clinical follow-up for up to two years after fract+ure or to completion of growth is necessary. The therapeutic concepts in cases of internal knee injuries such as intraligamentary cruciate ligament ruptures and patella luxations have undergone major changes in the past few years. In order to avoid instabilities and late sequelea surgical replacement of the anterior cruciate ligament is also performed in children with open growth plates. For patella luxations, both primary and secondary medial stabilisations have become accepted as further procedures for suture and reconstruction of the MPFL (medial femoro-patellar ligament).

\section{Einleitung}

Die Therapie von Verletzungen im Kindesalter erfolgte in der Vergangenheit vorwiegend konservativ. Allerdings stiegen mit der Zeit die Ansprüche an das Therapieergebnis sowie an die Mobilität.

OP-JOURNAL 2010; 26: 98-106

(c) Georg Thieme Verlag KG Stuttgart · New York DOI http://dx.doi.org/10.1055/s-0030-1250173
Je nach Lokalisation der Fraktur im Knochen sowie nach Richtung der Fehlstellung sind Spontankorrekturen von verbleibenden Fehlstellungen durch das weitere Wachstum möglich. Vor allem bei Frakturen in der Nähe der wachstumspotenten Epiphysenfugen in der Nähe des Kniegelenks besteht diese Möglichkeit [1,2]. Allerdings kommt es je nach Ausmaß der nötigen Korrektur zu einer Wachstumsstimulation mit der Gefahr der Beinverlängerung und daraus resultierender Beinlängendifferenz mit Einfluss auf die ganze Statik der Wirbelsäule und des Beckens. Aus diesem Grund plant man im deutschsprachigen Raum keine Spontankorrekturen von größeren Fehlstellungen standardmäßig in das Therapieregime mit ein.

In der Behandlung von Verletzungen der unteren Extremität müssen grundsätzlich die Schaftfrakturen (Diaphyse und Epiphyse) von den Gelenkfrakturen (Epi- 


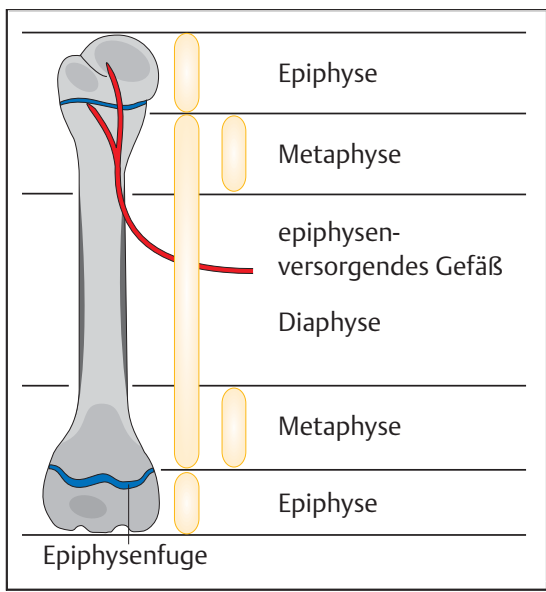

Abb. 1 Zonen des langen Röhrenknochens: Epiphyse, Metaphyse und Diaphyse.

physe) unterschieden werden (Abb. 1). Im Folgenden soll auf die wesentlichen Frakturen der unteren Extremität eingegangen werden. Bei den Verletzungen des Bandapparats des Kniegelenks werden die im Vordergrund stehenden Verletzungen des vorderen Kreuzbands und die Patellaluxation besprochen.

\section{Schaftfrakturen}

Femur

Femurschaftfrakturen sind selten. Meist liegt ein Hochenergietrauma zugrunde, weshalb weitere Verletzungen ausgeschlossen werden sollten. Bei Säuglingen und Kleinkindern (ca. bis zum 3. Lebensjahr) wird nach wie vor vorwiegend eine konservative Therapie im Beckenbeingips oder aber bei größerer Dislokation in der Overhead-Extension durchgeführt. Bei älteren Kindern ist in aller Regel eine mehrwöchige Immobilisation im Beckenbeingips auch bei gering dislozierten Frakturen den Patienten, aber auch dem sozialen Umfeld kaum noch zumutbar. Hier erfolgt in aller Regel eine geschlossene Reposition und osteosynthetische Versorgung. Nahezu alle Frakturen im Bereich des Femurschafts können mittels der elastischen intramedullären Nagelung (ESIN; Elastic Stable Intramedullary Nailing) versorgt werden. Verwendet werden hierzu elastische Titannägel, welche je nach Hersteller unterschiedliche Bezeichnungen haben (TEN [Synthes ${ }^{\circledR}$ ], ECMES, Nancy Nagel etc.). Im Bereich der Diaphyse stützt sich die ESIN auf das Prinzip der 3-Punkt-Abstützung durch 2 gegenläufig in den Markraum eingebrachte leicht vorgebogene Titannägel (Abb. 2). Bei korrekter Technik erlangt man hierdurch eine axiale und Rotationsstabilität sowie bei den Quer-
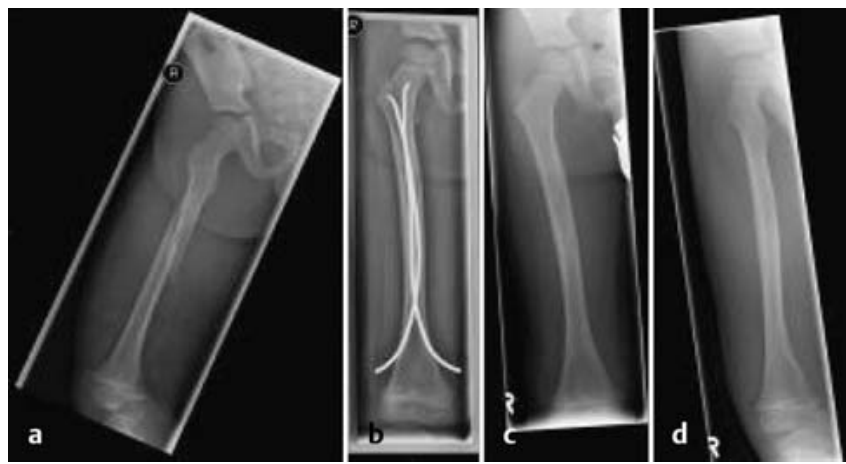

Abb. 2 a bis $d$

Femurschaftfraktur eines 5-jährigen Mädchens. Geschlossene Reposition und elastische intramedulläre Nagelung (ESIN).

frakturen eine Längsstabilität. Bei langen Schrägfrakturen ist nun noch eine Längsverschiebung der Fragmente um die Nägel herum mit Verkürzung möglich (Teleskoping). Durch Einbringen der sog. Endkappen (Synthes) und damit Verankerung der Nagelenden in der Metaphyse des Knochens können auch instabile Schrägfrakturen längsstabil mittels ESIN versorgt werden. Die Nägel können je nach Lokalisation der Fraktur retrograd (mittleres und proximales Drittel des Femurs) oder aber antegrad (distales Drittel des Femurs) eingebracht werden (Abb. 2 und 3). Diese Osteosynthese erlaubt Mikrobewegungen im Bereich der Fraktur, was zu einer schnellen Kallusbildung und Knochenbruchheilung führt. Der Vorteil dieser Methode liegt darin, dass die Wachstumsfugen im Vergleich zum intramedullären Nagel des Erwachsenen nicht tangiert werden. Sie ist kosmetisch günstig, da die Nägel über jeweils eine kleine Hautinzision eingebracht werden können. Aber auch metaphysäre proximale oder distale Frakturen können am Femur mittels ESIN versorgt werden über die Verankerung der Nägel in der metaphysären Spongiosa.

Technik ESIN am Bsp. der Femurfraktur [3]

\section{Material und Lagerung}

- Bildwandler, Bein frei beweglich abdecken

- intramedulläre elastische Titannägel (z.B. TEN; Synthes ${ }^{\circledR}$ ) in den Stärken $1,5-5 \mathrm{~mm}$

\section{OP-Technik}

- Zugang retrograd: Unter BV-Kontrolle Aufsuchen des Eintrittspunkts über der medialen und lateralen Metaphyse proximal der Wachstumsfuge auf gleicher Höhe

- Zugang antegrad: Unterhalb des Trochanter major am proximalen latera-

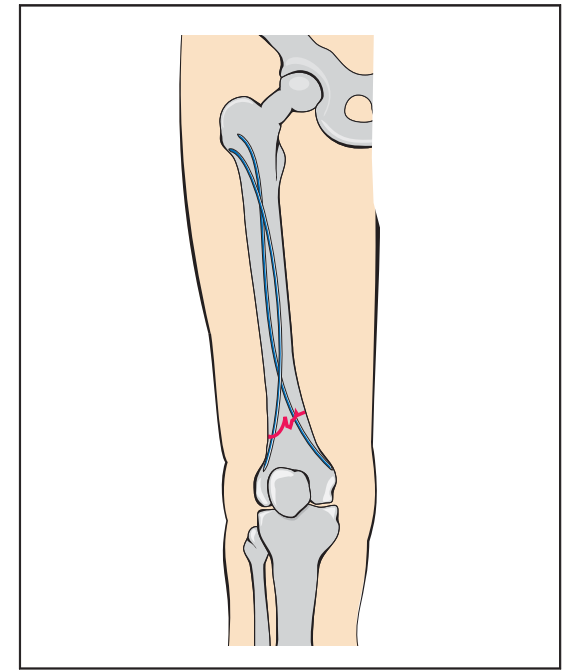

Abb. 3 Antegrade ESIN-Technik bei der distalen Femurschaftfraktur; aufgrund des unilateralen Zugangs muss ein Nagel s-förmig vorgebogen werden.

len Femur. 2. Eintrittspunkt ca. $2 \mathrm{~cm}$ distal davon

- Bestimmung der Nageldicke: 1/3 Markraumdurchmesser, Verwendung nur von gleich dicken Nägeln, ansonsten Gefahr eines Achsfehlers aufgrund der asymmetrischen Aufspannung der Nägel

- Vorbiegen der Nägel um ca. das 3-Fache des Markraumdurchmessers auf der Länge des Femurs (Aufspannen der Nägel im Markraum auf Höhe der Fraktur [3-Punkt-Abstützung])

- Bei der antegraden Technik muss ein Nagel s-förmig gebogen werden, damit es zu einer Aufspannung im Frakturbereich kommt (Abb. 3)

- Einbringen der Nägel bis zur Fraktur

- Reposition der Fraktur möglichst geschlossen, ggf. bei Weichteilinterposition über eine kleine Inzision über der Frakturstelle

- Vorschieben der Nägel bis auf ca. $1 \mathrm{~cm}$ in die prox. Metaphyse bzw. in den Schenkelhals nach Prüfung der Rotation (durch Vergleich der Innen- und 
Außenrotationsfähigkeit mit der Gegenseite)

- Kürzen der Nägel mit dem dafür vorgesehenen Instrumentarium und Vorschlagen der Nagelenden in den proximalen Femur mit dem entsprechenden Stößel, welcher eine Aussparung besitzt, damit der Nagel nicht zu weit in den Markraum eingeschlagen werden kann

- ggf. Aufsetzen und Eindrehen der Endkappen

- Bildwandlerkontrolle, Spülung und Wundverschluss

Nachbehandlung

- Querfrakturen der Femurdiaphyse können grundsätzlich schmerzabhängig belastet werden. Erfahrungsgemäß dauert es jedoch 3-6 Wochen bis das Kind aufgrund von Schmerzen oder Angst die Fraktur voll belastet. Eine Einschränkung der Beweglichkeit gibt es nicht

- Schrägfrakturen, proximale und distale metaphysäre Frakturen können je nach Alter des Kindes nach 4-6 Wochen nach Frakturheilung belastet werden

- Metallentfernung nach 4-6 Monaten

\section{Spezielle Komplikationen}

- Die häufigste Komplikation ist die Weichteilirritation durch zu lang belassene Nagelenden [4]. Zum einen ist auf die adäquate Kürzung zu achten, zum anderen lässt sich diese Komplikation durch Verwendung der Endkappen reduzieren.

- Sekundäre Dislokation; Instabilität; verzögerte Frakturheilung: Eine verzögerte Frakturheilung resultiert in der Regel aus einer nicht genügend stabilen Osteosynthese. Meist liegt eine Missachtung der Grundprinzipien der ESIN zugrunde (s. o.) [5].

Behandlungsalternativen

- Fixateur externe: bei ausgedehntem Weichteilschaden, instabiler Mehrfragmentfraktur, Polytrauma

- Platte: minimalinvasiv eingebrachte winkelstabile Platten bei ausgedehnter Trümmerzone, besonderen Indikationen

\section{Unterschenkel}

Die Unterschenkelfraktur ist die häufigste Fraktur bei Kindern an der unteren Extremität. In 70\% der Fälle liegt eine

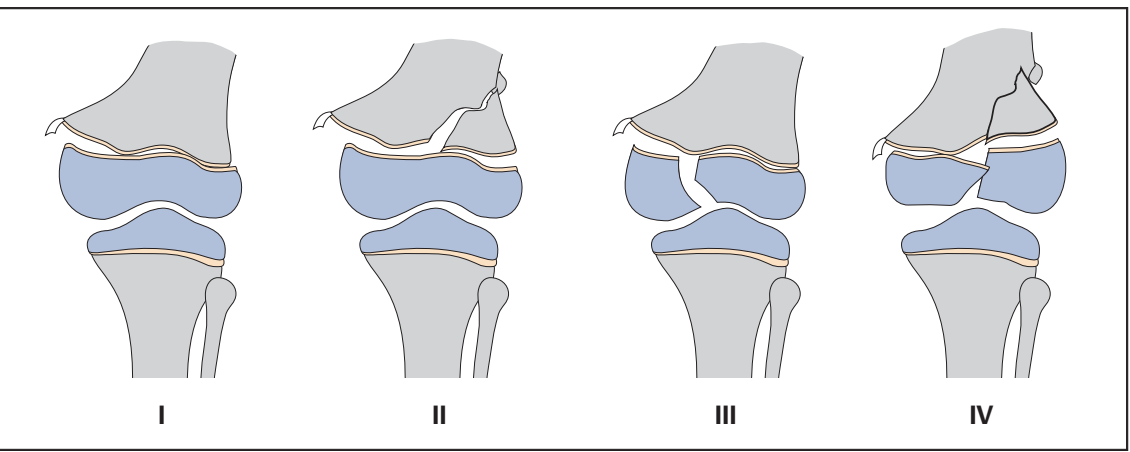

Abb. 4 Klassifikation der Frakturen der Wachstumsfuge nach Salter und Harris (modifiziert) oder Aitken.

isolierte Tibiafraktur vor. Diese gilt grundsätzlich als stabil und kann konservativ im Gips therapiert werden. Aufgrund des Muskelzugs bei noch intakter Fibula kommt es jedoch oft zu einer zunehmenden Varusfehlstellung. Eine Gipskeilung kann dieser Varusneigung entgegenwirken. Alternativ kann eine geschlossene Reposition und Osteosynthese erfolgen. Vollständige Unterschenkelfrakturen kommen in ca. 30\% vor. Sie gelten als instabil und neigen bei fehlender sperrender Wirkung der Fibula zur Valgusfehlstellung. Eine konservative Therapie ist z.T. möglich, birgt jedoch die Gefahr der Sekundärdislokation im Gips und ist oft für den Patienten schmerzhaft und unkomfortabel, sodass wir die Indikation zur Osteosynthese sehen [6]. Diese erfolgt entweder mittels antegrader ESIN oder Fixateur externe. Die Plattenosteosynthese findet in nur wenigen Ausnahmefällen Anwendung.

Material, Lagerung und Technik (Grundprinzipien ESIN: siehe Femurfraktur)

- Zugang: mediale und laterale proximale Tibiametaphyse, distal der Wachstumsfuge

- Fixateur externe: Verwendung eines monolateralen Fixateursystems

Verletzung der Tuberositas tibiae vermeiden. Die regelrechte Aufspannung der Nägel ist erschwert aufgrund der dreieckigen Form der Tibia. Die Nagelenden sollten nach dorsal gedreht werden, um die physiologische Antetorsion wiederherzustellen.

\section{Spezielle Komplikationen}

- ESIN: Weichteilirritationen durch zu lange Nagelenden bei geringem Weichteilmantel am proximalen Unterschenkel
- Fixation der Fraktur in Distraktion; zur Vermeidung Fraktur einstauchen

- Pintrack-Infektionen

\section{Metaphysäre Frakturen}

\section{Distaler Femur}

Wir unterscheiden hier die distale metapyhsäre Fraktur, welche selten auftritt, von der Fugenlösung mit oder ohne metaphysären Keil (Salter I und II; Aitken 0 und I; Abb.4), welche häufiger vorkommt. Unfallursache ist oft ein Hochenergietrauma, sodass Verletzungen des Gefäß-Nerven-Bündels in unsicheren Fällen ggf. mittels Angiografie ausgeschlossen werden müssen. Bei Säuglingen und Kleinkindern treten diese Verletzungen nicht selten auch im Rahmen einer Kindesmisshandlung auf, woran bei unklarer Anamnese gedacht werden sollte. Fehlstellungen nach dorsal (Retrokurvation) können bis zu einem gewissen Maß durch das weitere Wachstum korrigiert werden, andere Fehlstellungen sollten nicht belassen werden.

\section{Konservative Therapie}

Stabile Frakturen, v.a. Stauchungsfrakturen der distalen Femurmetaphyse, und undislozierte Frakturen können konservativ im Oberschenkelgips für 4-6 Wochen therapiert werden.

\section{Operative Therapie}

Instabile und dislozierte Frakturen müssen in Allgemeinanästhesie reponiert und fixiert werden. Bei metapyhsären Frakturen kann ggf. noch eine intramedulläre Fixation über antegrade ESIN erfolgen (s.o.). Ansonsten findet die perkutane gekreuzte Kirschner-Draht-Spickung ihre Anwendung, welche jedoch eine zusätzliche Gipsimmobilisation erfordert (Abb.5). Nach Frakturheilung 


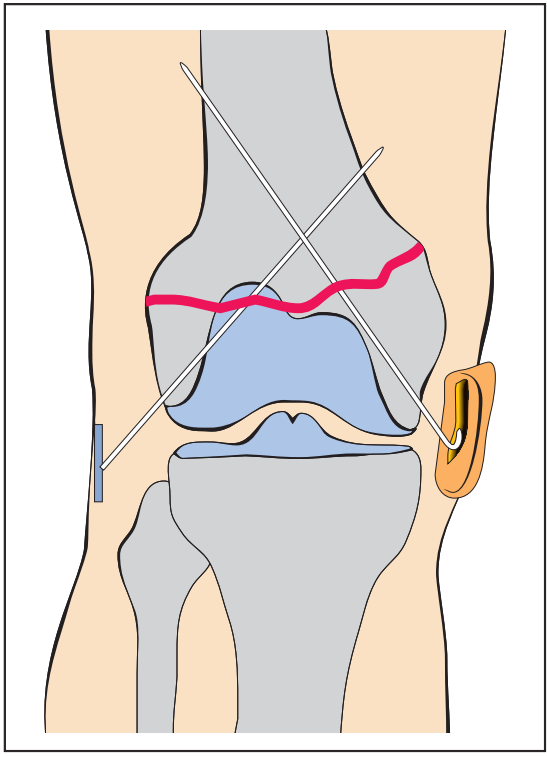

Abb. 5 Perkutane Kirschner-Draht-Spickung der Wachstumsfugenlösung (Salter II) am distalen Femur.

nach 4-6 Wochen können die Drähte ambulant entfernt und das Bein schmerzabhängig aufbelastet werden [7].

\section{Proximaler Unterschenkel}

Man unterscheidet die unkomplizierten metaphysären Stauchungsfrakturen von den Biegungsfrakturen, welche zu einer zunehmenden Valgusfehlstellung neigen, und den Fugenlösungen. Auch hier muss stets aufgrund der unmittelbaren Lage der Gefäße zur Frakturregion an eine begleitende Gefäßverletzung gedacht werden.

\section{Konservative Therapie}

Undislozierte stabile Frakturen werden im Oberschenkelgips für 4-6 Wochen behandelt.

Die Biegungsfraktur der proximalen Tibiametaphyse neigt aufgrund einer einseitigen Wachstumsstimulation zu einer zunehmenden Achsfehlstellung mit Ausbildung einer Genu valgum (X-Bein). Aus diesem Grund gilt bereits ein sichtbarer Frakturspalt als Dislokation. Die mediale Frakturzone muss unter Kompression gebracht werden, konservativ mittels Gipskeilung oder operativ.

\section{Operative Therapie}

Dislozierte und instabile Frakturen werden in Allgemeinanästhesie reponiert und fixiert. Dies erfolgt vorwiegend durch eine perkutane gekreuzte Kirsch-

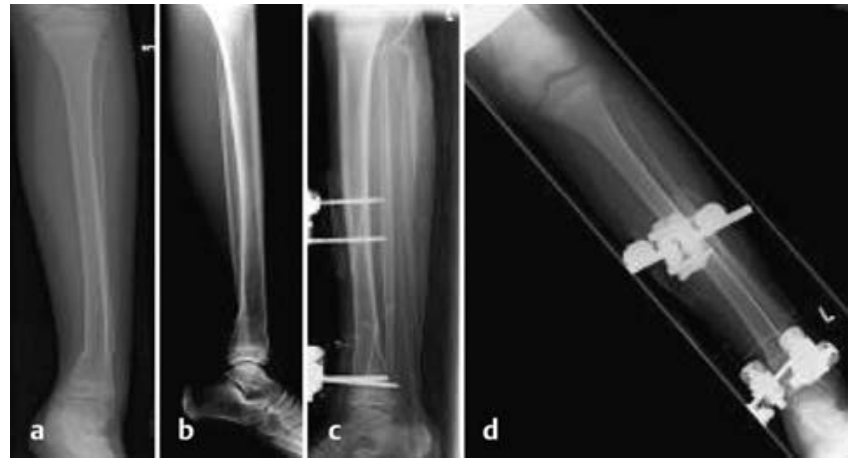

Abb. 6 a bis d Spiralfraktur der distalen Tibia eines 10jährigen Mädchens. Stabilisierung mittels Fixateur externe.
ner-Draht-Osteosynthese. Bei der proximalen Biegungsfraktur stehen entweder eine Plattenosteosynthese oder ein medialer Fixateur externe zur Verfügung, um entsprechend Kompression auf den medialen Frakturspalt zu erlangen.

\section{Distaler Unterschenkel}

Stauchungsfrakturen treten häufig im Rahmen sog. „Fahrradspeichenverletzungen“ auf und gelten i.d.R. als stabil. Biegungsbrüche in diesem Bereich neigen wie an der proximalen Metaphyse auch durch eine einseitige Wachstumsstimulation zu Achsfehlern, sodass größere Achsfehlstellungen nicht belassen werden sollten. Meist liegt eine Rekurvationsfehlstellung vor, welche durch das weitere Wachstum bis zu einem gewissen Grad korrigiert werden kann. Die Fugenlösungen gehören am distalen Unterschenkel zu den häufigsten Verletzungen, meist infolge eines Umknicktraumas. Distale Fibulafrakturen, wie wir sie vom Erwachsenen kennen, sind selten beim Kind. Meist liegt eine begleitende Fibulafraktur im Rahmen eine Fugenlösung der distalen Tibia vor. Bei intaktem Band- und Syndesmosenapparat ist i.d.R. eine Reposition und Stabilisierung der tibialen Fugenlösung ausreichend.

\section{Konservative Therapie}

Undislozierte stabile Frakturen werden im Unterschenkelgips für 4-6 Wochen behandelt. Durch eine leichte Spitzfußstellung des Gipses kann der Rekurvation zu einem gewissen Maß entgegengewirkt werden.

\section{Operative Therapie}

Dislozierte Frakturen werden in Allgemeinanästhesie reponiert und bei fehlender Stabilität fixiert. Die metaphysären Frakturen können mittels perkutaner gekreuzter Kirschner-Draht-Spickung oder durch Anlage eines Fixateur externe stabilisiert werden (Abb.6). Eine intramedulläre Osteosynthese mittels ESIN gelingt meist nicht mit ausreichender Stabilität. Fugenlösungen können, wenn nach Reposition stabil, im Unterschenkelgips weiterbehandelt werden. Oft „rutscht“ die Fraktur nach der Reposition jedoch wieder in die Ausgangsposition zurück, sodass dann eine gekreuzte Kirschner-Draht-Spickung durchgeführt werden muss. Bei ausreichend großem metaphysären Keil kann auch eine fugenparallele Schraubenosteosynthese erfolgen. Eine offene Reposition mit Entfernung von eingeschlagenem Periost ist in aller Regel nicht notwendig. Nur bei unmöglicher Reposition muss ein offenes Vorgehen gewählt werden.

\section{Gelenkfrakturen}

Bei Frakturen der Epiphyse ist zum einen die Wachstumszone der Epiphysenfuge, zum anderen aber auch die Gelenkfläche betroffen. Ziel ist eine anatomische Rekonstruktion der Gelenkfläche, um einer frühzeitigen Arthroseentwicklung vorzubeugen und eine möglichst optimale Reposition, um die Gefahr der hemmenden Wachstumsstörung (s.u.) zu reduzieren [8]. Man unterscheidet epiphysäre Frakturen bei noch weit offenen Wachstumsfugen (sog. Salter-III- und -IV-Frakturen; Abb.4) von den epiphysären Frakturen bei Adoleszenten bei bereits begonnenem Fugenschluss (sog. Übergangsfrakturen). Im letzteren Fall kann das Risiko einer relevanten Wachstumsstörung vernachlässigt werden bei bereits teilweise verschlossener Wachstumsfuge. Gelenkfrakturen treten deutlich seltener auf als Schaftfrakturen. Am Hüftkopf sind sie eine Rarität. Auch im Bereich des Kniegelenks sind sie selten, hier sollte stets an die Möglichkeit intraartikulärer Begleitverletzungen gedacht werden. Am häufigsten mit in ca. 25\% der Sprunggelenksverletzungen treten Epiphysenfrakturen an der distalen Tibia auf. 


\section{Konservative Therapie}

Alle Frakturen ohne Gelenkstufe und eine Dehiszenz $<2 \mathrm{~mm}$ können konservativ durch Immobilisation therapiert werden. Eine radiologische Stellungskontrolle nach 5-8 Tagen sollte zur Erkennung einer Sekundärdislokation durchgeführt werden.

\section{Operative Therapie}

Ziel ist eine anatomische Gelenkrekonstruktion durch offene oder arthroskopisch gestützte Reposition der Fraktur und Fixation durch eine Schraubenosteosynthese. Hierbei sind möglichst selbstschneidende Schrauben mit kurzem Gewinde $\mathrm{zu}$ verwenden, um eine iatrogene Fugenverletzung durch die Hitzeentwicklung beim Bohren zu vermeiden und Kompression auf den Frakturspalt zu erlangen. Die Schrauben müssen bei noch offenen Wachstumsfugen parallel zu Fuge und Gelenk eingebracht werden, um die Wachstumszone nicht zu verletzen. Bei den Übergangsfrakturen kann dies vernachlässigt werden.

Die Wachstumsfuge beginnt sich an der distalen Tibia von medial nach lateral über einen Zeitraum von 18 Monaten langsam zu verschließen. Je nach Stadium des Fugenschlusses kommt es bei der Übergangsfraktur zu einem Ausbrechen eines noch nicht verschlossenen ventrolateralen epiphysären Anteils. Liegt nur die ventrolaterale epiphysäre Fraktur vor im Sinne eines knöchernen Syndesmosenausrisses, so spricht man von der Twoplane- oder auch nach dem Erstbeschreiber Tillaux-Fraktur. Wirken zusätzliche Torsionskräfte ein, so kann es zu einem Ausbrechen eines dorsalen Keils, vergleichbar mit dem VolkmanDreieck beim Erwachsenen, kommen, die sog. Triplane-Fraktur.

Bei bereits weit fortgeschrittenem $\mathrm{Fu}-$ genschluss lässt sich eine adäquate Reposition des ventrolateralen epiphysären Fragments oft nur durch einen schrägen Schraubenverlauf gewährleisten (Abb. 7).

Je nach Stabilität und Compliance des Patienten kann eine gipsfreie Nachbehandlung erfolgen unter Abrollbelastung für 4-6 Wochen. Die Metallentfernung wird nach 3 Monaten durchgeführt.
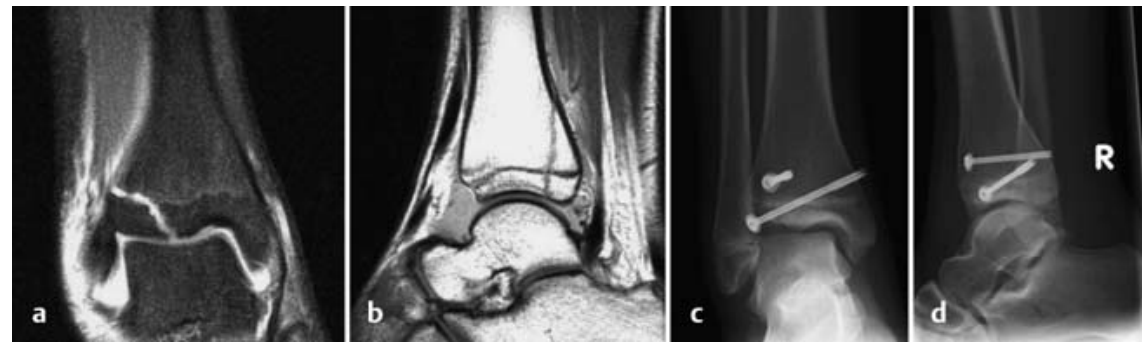

Abb. 7a bis d Triplane-II-Fraktur eines 12-jährigen Mädchens (MRT) mit nahezu vollständigem Wachstumsfugenverschluss. Offene Reposition und Osteosynthese mittels epiphysärer „fugenkreuzender" und metaphysärer Zugschraube.

\section{Komplikationen}

\section{Stimulative Wachstumsstörung}

Nach allen Frakturen kommt es zu einer gewissen Wachstumsstimulation. Das Ausmaß ist von der Wachstumspotenz der nahe liegenden Fuge sowie der verbleibenden Fehlstellung und damit der nötigen Spontankorrektur abhängig (auch s.o.) [7].

\section{Hemmende Wachstumsstörungen}

Durch Verletzung der Wachstumsfuge entweder direkt oder über Läsion des versorgenden Gefäßsystems kann es zu einem vorzeitigen Fugenverschluss kommen. Ein vollständiger Verschluss mit nachfolgender Beinverkürzung ist selten. Häufig kommt es jedoch zu einem partiellen Fugenverschluss mit nachfolgendem Fehlwachstum und Achsfehlstellung, je nach Lokalisation und Verletzung in bis zu 50\% der Fälle [9,10]. Das Risiko steigt mit der Nähe der Fraktur zur Wachstumsfuge und dem Dislokationsgrad. Über diese Möglichkeit sind die Eltern und der Patient im Vorfeld aufzuklären. Diese Wachstumsstörungen sind nicht vorherzusehen oder zu vermeiden, allerdings kann das Risiko durch Vermeidung wiederholter Repositionsmanöver und durch eine möglichst optimale Reposition vermindert werden. Aus diesem Grund sollten die Kinder bis zu 2 Jahre nach dem Unfall bzw. bis zum Wachstumsabschluss in klinischer Nachkontrolle verbleiben, um relevante Wachstumsstörungen rechtzeitig zu erkennen und behandeln zu können.

\section{Pseudarthrosen}

Eine verzögerte oder ausbleibende Frakturheilung ist beim Kind am Ellenbogen bekannt, an der unteren Extremität jedoch sehr selten. Auch Frakturen in deutlicher Dislokationsstellung heilen stabil aus. Tritt eine verzögerte Frakturheilung

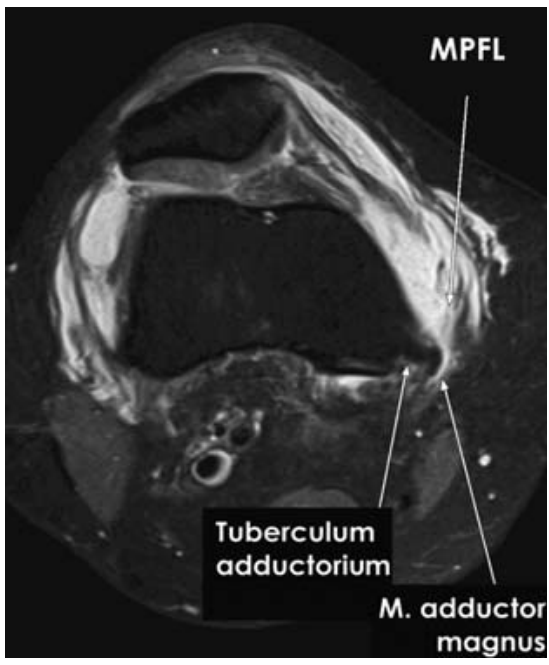

Abb. 8 MR-Tomografie einer Ruptur des MPFL bei Z.n. stattgehabter Patellaluxation.

ein, so muss meist an eine inadäquate Osteosynthese mit verbleibender Instabilität gedacht werden.

\section{Patellaluxation/Patellainstabilität}

Patellaluxationen treten mit einer Inzidenz von 43-77/100000/Jahr relativ häufig auf. Es findet sich ein typischer Altersgipfel in der Adoleszenz zwischen dem 10. und 17. Lebensjahr. Das weibliche Geschlecht ist dabei deutlich häufiger betroffen als das männliche. Grundsätzlich muss bei der Patellaluxation die primär traumatische und anschließend häufig rezidivierende Patellaluxation von der mit 90\% am häufigsten auftretenden atraumatischen, habituellen Patellaluxation unterschieden werden $[11,12]$.

Die Patella entwickelt sich ab der 7. Embryonalwoche als kartilaginäre Anlage innerhalb der Patellarsehne. Ihre Ossifikation beginnt zwischen dem 4. und 7. Lebensjahr. Es entstehen eine mediale und laterale Facette, die bei regelrechter Passform mit der Trochlea korrespondiert [13]. Bei Beugung des Kniegelenks 
entsteht zwischen 20 und $90^{\circ}$ Flexion ein patellofemoraler Kontakt, d.h. wenn die Patella in die Trochlea eintaucht. Daraus lässt sich ableiten, dass eine Patellaluxation eher in Extensionsnähe entsteht und zwar entweder durch eine direkt einwirkende Kraft oder durch einen entsprechend nach lateral gerichteten Kraftvektor bzw. durch eine plötzliche Kontraktur des M. quadrizeps in Beugestellung des Kniegelenks [1, 14,15].

Da der traumatischen Luxation ein adäquates Trauma zugrunde liegt, führt ein Fortbestehen der Pathologie häufig zu einem Rezidiv. Demgegenüber liegen bei der atraumatischen Patellaluxation verschiedene prädisponierende Faktoren zugrunde. Begünstigend für eine Patellainstabilität sind

- Trochleadysplasie,

- Patelladysplasie,

- ein pathologischer Q-Winkel,

- Patella alta,

- Genu valgum,

- vermehrte Innenrotation [16,17].

Kommt es bei adäquatem Trauma zu einer Luxation der Patella, so können verschiedene intraartikuläre Pathologien, wie „bone bruise“ der medialen Patella und des lateralen Femurkondylus, Abriss des medialen Retinakulums und/ oder des medialen femoralen Ligaments, aber auch osteochondrale Abscherfrakturen auftreten. Klinisch fallen der Druckschmerz im Bereich der medialen Patellafacette und des medialen Halteapparats (ggf. auch eine tastbare Delle) sowie der intraartikuläre Erguss auf. Der Apprehension-Test ist in der Regel deutlich positiv. Neben der nativradiologischen Abklärung ist im Weiteren die Durchführung einer MRT indiziert. Hierbei können Verletzungen des medialen patellofemoralen Komplexes sowie osteochondrale Frakturen objektiviert werden. Bei einer chronischen Instabilität und dem Vorliegen einer Dysplasie ist die CT-Untersuchung hilfreich, das genaue Ausmaß der kausalen Pathologie einzuschätzen [18].

Der mediale patellofemorale Komplex dient der Patellaführung in die Trochlea und verhindert eine Luxation der Patella nach lateral. $\mathrm{Zu}$ den medialen Stabilisatoren zählen der M. vastus medialis als dynamischer Stabilisator, das mediale patellofemorale Band als passiver Stabilisator und das mediale tibiopatellare und meniskopatellare Band, welche hauptsächlich propriozeptive Funktionen haben [19-21].

\section{Therapie}

\section{Konservative Therapie}

Erstereignisse ohne begleitende Knorpelschäden stellen die klassische Indikation zur konservativen Behandlung dar. Zunächst erfolgt, falls nicht bereits spontan reponiert, die Reposition der Patella. Anschließend wird das betroffene Kniegelenk zunächst für 1 Woche in $10-20^{\circ}$ Flexionsstellung ruhig gestellt. In der 2 . und 3. Behandlungswoche wird der Bewegungsumfang in einer entsprechenden Kniegelenksorthese auf Flex./Ext. 60-20- $0^{\circ}$ beginnend freigegeben und schließlich in der 4.-6. Woche auf Flex./ Ext. 90-10-0 $0^{\circ}$ erweitert. Der Übergang zur Vollbelastung erfolgt nach vollständigem Rückgang des intraartikulären Kniegelenksergusses.

\section{Operative Therapie}

Neben dem konservativen Vorgehen mit initialer Ruhigstellung des Kniegelenks und einem restriktiven Belastungsaufbau sind in der Vergangenheit unzählige Operationsverfahren beschrieben worden. Bei den Langzeitergebnissen zeigt sich, dass die Rezidivrate nach konservativem Vorgehen bis zu 63\% beträgt, während bei Patienten, die operativ behandelt wurden, die Reluxationsrate zwischen 7 und 25\% liegt.

Bei den Operationsverfahren werden Weichteileingriffe und knöcherne Eingriffe unterschieden. Die Weichteileingriffe reichen vom einfachen lateralen Release und einer medialen Raffung [22, 23] über Eingriffe am M. quadriceps (nach Insall), Distalisierung des M. vastus medialis (nach Madigan), Sehnentransfers (Gracilistransfer nach Lanz oder Witt; Semitendinosustransfer nach Galeazzi oder nach Dewar) [24,25]. Aber auch Eingriffe wie der Transfer einer Patellarsehnenhälfte (nach Goldwaith) sowie die Interposition eines Retinakulumstreifens (nach Krogius) sind mit unterschiedlichem Outcome beschrieben worden [26-28]. Zu den knöchernen Eingriffen zählen Verfahren wie der Tuberositas-Transfer (nach Emslie-Trillat oder nach Roux), die suprakondyläre Umstellungsosteotomie und in den letzten Jahren die Trochleaplastik [29,30, 38]. Osteotomien im Bereich der Patella haben sich aufgrund der Komplikationsrate nicht durchgesetzt [37].
Operationsindikation

Die Operationsindikation nach primär traumatischer Patellaluxation wird in der Literatur kontrovers diskutiert. Einigkeit besteht hinsichtlich der Fälle, bei denen zusätzlich chondrale oder osteochondrale Läsionen vorliegen. Hierbei erfolgt je nach Lokalisation der Läsion innerhalb des medialen patellofemoralen Komplexes (intrasubstanziell, patellanah oder am femoralen Ursprung) eine Refixation mit entsprechenden Fadenankersystemen oder die primäre Naht. Bei persistierendem Apprehensionzeichen oder $>2$ Luxationsereignissen und/oder massiver Patellarlateralisation bevorzugen die Autoren eine Rekonstruktion des Lig. patellofemorale mediale (MPFL), ggf. in Kombination mit einer additiven Medialisierung der Tuberositas tibiae im Falle eines TT-TG-(Tuberositas tibiae-Tibial groove-)Abstands von > $15 \mathrm{~mm}$.

\section{MPFL-Plastik}

Das MPFL ist der wichtigste statische Stabilisator der Patella (Abb. 8) [31]. Die Rekonstruktion des MPFL erfolgt mittels Gracilisplastik. Hierbei wird zunächst über eine etwa $2-3 \mathrm{~cm}$ lange Hautinzision im Verlauf des Pes anserinus die Sehne des M. gracilis entnommen. Die Sehne wird anschließend von dem verbliebenen Muskelgewebe befreit und an beiden Enden mit einem resorbierbaren Faden auf einer Länge von $10 \mathrm{~mm}$ armiert. Zur Darstellung der Insertionsstelle des MPFL wird medial über dem proximalen Drittel der Patella ebenfalls ein $2 \mathrm{~cm}$ langer Hautschnitt gelegt und die superomediale Patellarkante dargestellt [32]. Zur Fixation des Grafts werden 2 KDrähte, einer ganz proximal und der 2 . etwa $2 \mathrm{~cm}$ distal davon, tangential in die Patella vorgebohrt und schließlich mit einem kanülierten 4-mm-Bohrer überbohrt. Die amierten Enden des Grafts können so mittels Swivelock-Anker (Fa. Arthrex) in die Patella eingebracht und fixiert werden (Abb. 9) [33].

Anschließend wird die 2. Schicht des medialen patellofemoralen Komplexes aufgesucht und dabei sorgfältig die Gelenkkapsel geschont. Mit einem Overholt erfolgt anschließend die stumpfe Präparation innerhalb der 2. Schicht Richtung femoraler Insertionsstelle zwischen Tuberculum adductorium und Epicondylus medialis. In leichter Flexionsstellung wird sodann über der Spitze des Overholts eine kurze längsverlaufende Hautinzision angelegt und ein Faden durch- 


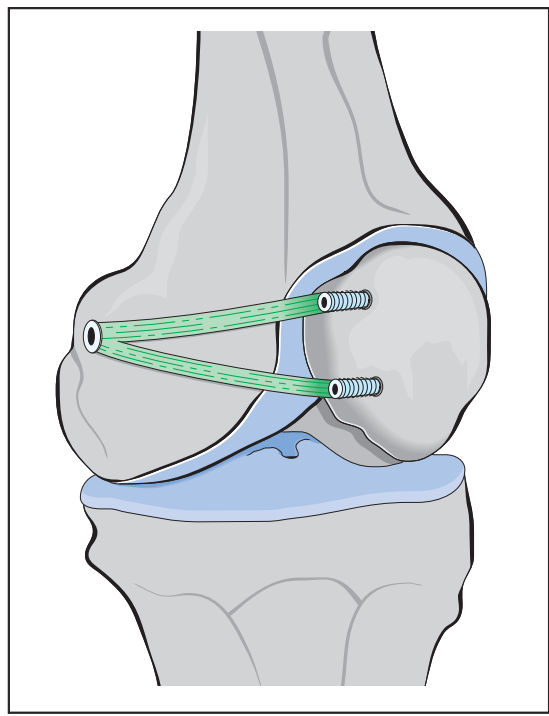

Abb.9 Schematische Darstellung der MPFLPlastik mittels Gracilistransplantat. Die patellare Verankerung erfolgt über z.B. 2 Anker. Femoral wird die Gracilissehne mit einer Biointerferenzschraube fixiert.

gezogen [32]. Die exakte femorale Insertionsstelle wird unter BV-Kontrolle in streng seitlicher Projektion mittels Draht markiert. Orientierungshilfen zur anatomischen Insertion femoral sind dabei die Verlängerung der posterioren Femurkortikalis sowie die Senkrechten durch den proximalen Ansatz des medialen Kondylus und den posteriorsten Punkt der Blumensaat-Linie (Abb. 10). Der korrekt platzierte Draht wird sodann mit einem Bohrer, der $1 \mathrm{~mm}$ größer als der Durchmesser des Grafts ist, überbohrt [33].

Über die vorgelegte Fadenschlinge kann im nächsten Schritt das Graft durch die 2. Schicht zur femoralen Insertion durchgezogen und schließlich in das femorale Bohrloch eingezogen werden. Die femorale Fixation erfolgt dann entsprechend des Bohrkanaldurchmessers mittels Biointerferenzschraube. Bei der Fixation in $30^{\circ}$ Flexionsstellung ist darauf zu achten, dass die laterale Patellafacette mit dem lateralen Kondylenrand abschließt.

Überkorrektur vermeiden, um einem Extensions- bzw. Flexionsdefizit vorzubeugen. Durch die Bohrungen in der Patella zur Transplantatfixierung kann diese geschwächt werden. Es sind in Einzelfällen Patellarfrakturen nach MPFL-Plastik beschrieben worden.

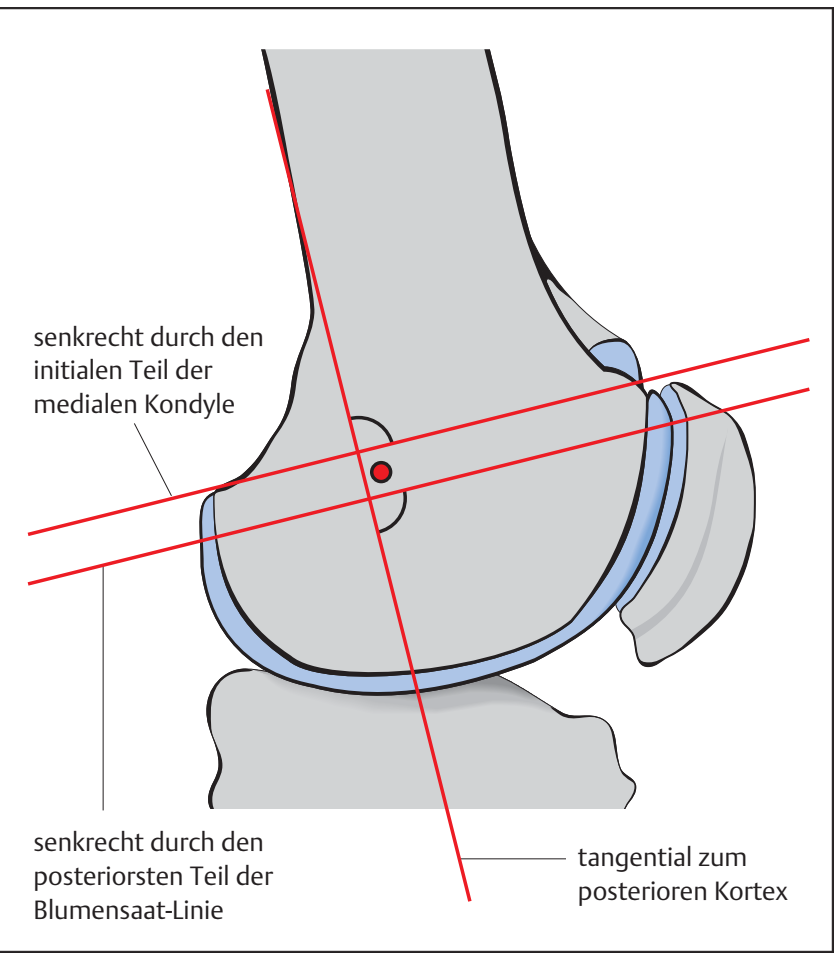

Abb. 10 Die anatomische Insertionsstelle der MPFL-Plastik wird femoral unter Durchleuchtung markiert. Als Orientierungshilfe dienen hierbei die Verlängerung der posterioren Femurkortikalis sowie die beiden Senkrechten durch (a) den hinteren Teil des medialen Femurkondylus und (b) den posterioren Teil der Blumensaat-Linie.

\section{Nachbehandlung}

Die Nachbehandlung erfolgt bis zum Abschluss der Wundheilung mit einer Teilbelastung von $20 \mathrm{~kg}$. Aktive Quadrizepsübungen sind ab dem 1 . postoperativen Tag gestattet. Der Bewegungsumfang wird mittels Kniegelenksorthese für jeweils 2 Wochen auf $30^{\circ}, 60^{\circ}$ und $90^{\circ}$ limitiert. Leichte sportliche Betätigung ist 6 Wochen postoperativ erlaubt, volle Sportfähigkeit ist frühestens nach 3 Monaten erlaubt [33].

\section{Vordere Kreuzbandruptur}

\section{Eminentia-intercondylaris-Ausriss}

Die klassische Verletzung des vorderen Kreuzbands (VKB) beim Kind mit offenen Wachstumsfugen besteht in einem knöchernen Bandausriss (Kinder bis zum 12. Lebensjahr: Eminentiafraktur), was auf die höhere Stabilität gegenüber den osteochondralen Strukturen in diesem Alter zurückzuführen ist. Isolierte intraligamentäre VKB-Rupturen treten eher bei Kindern ab dem 12. Lebensjahr auf und hängen mit der zunehmenden Stabilität der knöchernen Strukturen zusammen. Kombinationen von knöchernen und ligamentären Läsionen sind ebenfalls möglich. Dislozierte knöcherne Ausrisse des VKB lassen sich bereits nativradiologisch nachweisen. Im Falle eines nicht dislozierten knöchernen Aus- risses ist die MRT zielführend. Die Eminentiafraktur ist die häufigste epiphysäre Fraktur der proximalen Tibia. Sie liegt intraartikulär, ohne Beteiligung der Epiphysenfuge [34]. Die Einteilung erfolgt nach dem Dislokationsgrad nach Meyers und McKeever:

- Grad I: undisloziert (konservatives Vorgehen)

- Grad II: hängende Fraktur (geschlossene Reposition und Fixation bzw. arthroskopisch kontrollierte Reposition)

- Grad IIla: dislozierte Fraktur (arthroskopische Reposition und Fixation; ausnahmsweise offen)

- Grad IIIb: disloziert und verdrehte Fraktur (wie bei Grad IIIa)

Ab Grad II kann eine operative Reposition und entsprechende Fixation notwendig werden. Dabei muss insbesondere darauf geachtet werden, dass die Vorderhörner der Menisken nicht im Frakturspalt eingeklemmt sind und ein Repositionshindernis darstellen $[2,34]$.

\section{Intraligamentäre vordere Kreuzbandläsion}

Die intraligamentären Verletzungen des VKB treten vorwiegend in der Adoleszenz auf. Häufig liegen entsprechende Begleitverletzungen im Bereich der Menisci und Kollateralbänder vor. Ob die steigende Inzidenz der VKB-Rupturen im Adoleszentenalter auf ein geändertes Freizeitverhalten zurückzuführen ist 
oder der verbesserten Diagnostik mit entsprechend frühem Einsatz der MRT Rechnung trägt, bleibt abzuwarten. Genaue Zahlen zur Häufigkeit der VKBRuptur im Kindesalter liegen nicht vor. Tatsache ist, dass die vollständige intraligamentäre Ruptur weiterhin sehr selten auftritt. Mädchen sind häufiger betroffen als Jungen.

Ziel der Therapie ist die Beseitigung einer Kniegelenksinstabilität. Ein konservatives Vorgehen bei Kindern hat sich aufgrund mangelnder Compliance als nicht Erfolg versprechend erwiesen, da sich die Kinder in ihrer körperlichen Aktivität nur schlecht einschränken lassen.

Obgleich die Indikation zur operativen Versorgung der VKB-Ruptur im Kindesund Adoleszentenalter weiterhin kontrovers diskutiert wird, geht heute der Trend eindeutig zur operativen Versorgung mittels früher VKB-Ersatzplastik, auch bei noch offenen Wachstumsfugen. Zum einen werden so instabilitätsbedingte Meniskus- und Knorpelschäden mit der Gefahr der Früharthrose vermieden, zum anderen konnten verschiedene Untersuchungen bisher keine Wachstumsstörung aufgrund einer iatrogenen Schädigung der Epiphysenfuge nachweisen.

Die teils favorisierte intraligamentäre Naht des VKB bei Kindern unter 12 Jahren mit weit offenen Wachstumsfugen scheint in den Augen der Autoren trotz unterschiedlicher Ergebnisse in bestimmten Fällen noch gerechtfertigt. Eine sichere Ausheilung und Stabilität sollte in diesen Fällen gewährleistet sein, ansonsten ist eine VKB-Plastik durchzuführen.

\section{Operative Therapie}

Bei der Planung der VKB-Ersatzplastik im Kindesalter ist das biologische Alter zu berücksichtigen, da hiervon die Wahl der Operationstechnik abhängt. Es ist allgemeiner Konsens, dass Adoleszenten, die sich im letzten großen Wachstumsschub befinden oder diesen bereits hinter sich haben, im Grunde genommen wie Erwachsene behandelt und operiert werden können. Die Sorge, dass Wachstumsstörungen nach einer VKB-Ersatzplastik auftreten könnten, ist unbegründet. Gleiches gilt auch für Kinder mit weit offenen Wachstumsfugen (Tanner Stadium 1 und 2), wie neuere Studien zeigen konnten. Es wurden keine Beinlängendifferenzen, keine Wachstums- störungen und kein Fehlwachstum (varus oder valgus) beobachtet [35].

Die VKB-Plastik wird im Kindesalter immer transepiphysär, d.h. fugenkreuzend als 3- oder 4-fach gebündelte Semitendinosussehne in Ein-Kanal-Technik positioniert. Hierbei ist eine möglichst isometriegerechte Insertion tibial und femoral anzustreben. Je jünger der Patient, desto geringer sollte der Durchmesser des Knochenkanals ausfallen. Die Fixation der Transplantate erfolgt bei Kindern immer extrakortikal. Die Wachstumsfuge darf nicht von Fixationsmaterial oder Knochenblöcken gekreuzt werden. Begleitverletzungen (z.B. Meniskusläsionen) werden im gleichen Eingriff behandelt. Im Falle von Kombinationsverletzungen werden frische Seitenbandrupturen zuerst genäht $[35,36]$.

Nach Abschluss der diagnostischen Arthroskopie zum Ausschluss bzw. zur Therapie von Begleitverletzungen wird zunächst die Semitendinosussehne in üblicher Weise gewonnen. Über einen schrägen Hautschnitt im Verlauf des Pes anserinus wird die Sehne des M. semitendinosus mit einem geschlossenen Sehnenstripper entnommen. Die Sehne wird anschließend von dem verbliebenen Muskelgewebe befreit und je nach Durchmesser 3- bzw. 4-fach gelegt, an beiden Enden mit einem resorbierbaren Faden armiert. Der tibiale Bohrkanal sollte möglichst steil ausgerichtet werden, damit die tibiale Wachstumsfuge nahezu senkrecht getroffen und der Querschnitt der Fugenverletzung möglichst gering gehalten wird. Daher wird bei dem entsprechenden Zielgerät ein Winkel von $55-60^{\circ}$ in der Sagittalebene eingestellt und $45^{\circ}$ in der Frontalebene gebohrt, um den Rand der Apophyse nicht zu verletzen. Ferner ist dabei darauf zu achten, dass der Führungsdraht auf Höhe des Außenmeniskusvorderhorns austritt. Nach Überbohren des tibialen Führungsdrahts wird entweder transtibial oder über das anteromediale Portal der femorale Bohrkanal angelegt. Hierfür wird das femorale Zielgerät mit einer Unterstellung von 4-6 mm je nach Transplantatdurchmesser gewählt. Das Zielgerät wird bei 10:30 Uhr (rechtes Kniegelenk) bzw. 13:30 Uhr (linkes Kniegelenk) an der Hinterkante des lateralen Femurkondylus eingehängt. Anschließend wird der Drill Pin durch das Femur gebohrt, bis er die Haut des Oberschenkels perforiert. Nun erfolgt das Überbohren mit dem femoralen Kopfbohrer entsprechend dem Durchmesser des Trans- plantats, um ein etwa $35 \mathrm{~mm}$ tiefes Sackloch zu generieren. In die Öse des Drill Pin wird dann ein Shuttlefaden eingefädelt und über den Drill Pin schließlich aus dem Femur durch die Haut gezogen. Entsprechend der femoralen Bohrkanallänge und der gewünschten Insertionstiefe des Transplantats wird die Länge des Retro Button Loop berechnet (z. B. femorale Kanallänge $50 \mathrm{~mm}$ - gewünschte Insertionstiefe $25 \mathrm{~mm}=25 \mathrm{~mm}$ Retro Button Loop). Dabei ist darauf zu achten, dass das Sackloch ausreichend tief gebohrt wurde, damit beim Einziehen der Retro Button auch flippen kann (z. B. Retro Button Loop $25 \mathrm{~mm}+10 \mathrm{~mm}$ Zugabe zum Flippen $=$ Sacklochtiefe $35 \mathrm{~mm}$ ). Nach Einzug des Transplantats mithilfe des vorgelegten Shuttlefadens und flippen des Buttons femoral wird die Zugfestigkeit und regelrechte femorale Verankerung des Transplantats geprüft. Hierzu mehrmaliges Durchbewegen des Kniegelenks unter kontinuierlichem Zug. Unter arthroskopischer Sicht wird ebenfalls die korrekte Lage des Transplantats geprüft und kontrolliert, ob das Transplantat ohne Impingement in die Notch eintaucht. Schließlich wird in $30^{\circ}$ Beugestellung tibial das Transplantat fixiert, indem die nicht resorbierbaren Fäden über einer Suture Disc verknotet werden. Abschließende Kontrolle der vollen Streckung, Fasziennaht und Hautnaht.

\section{Nachbehandlung}

Die Nachbehandlung erfolgt bis zum Abschluss der Wundheilung mit einer Teilbelastung von $20 \mathrm{~kg}$. Konsequentes Tragen einer Kniegelenksorthese. Aktive und passive Bewegung ohne Widerstand in geschlossener Kette ab dem 2. postoperativen Tag. Beugung gegen Widerstand ab der 3. postoperativen Woche. Vermeiden der aktiven Streckung gegen Widerstand zwischen 0 und $30^{\circ}$ Flexion bis zur 12. postoperativen Woche. Fahrradfahren und Schwimmen ist ab der 6. postoperativen Woche gestattet. Jogging ab der 12 . postoperativen Woche, volle Sportfähigkeit ist frühestens nach 6 Monaten erlaubt.

Verletzung der Wachstumsfugen femoral und tibial können das Risiko einer Wachstumsstörung, insbesonder bei präpubertären Kindern in sich bergen. In der Folge der extrakortikalen Fixation besteht das Risiko einer Tunnelerweiterung. 


\section{Literatur}

${ }^{1}$ Schneidmueller D. Knochenbruchheilung. In: Weinberg AM, Schneidmueller D, Hrsg. Unfallchirurgie bei Kindern. Köln: Deutscher Ärzte Verlag; 2010

${ }^{2}$ Marzi I, Hrsg. Kindertraumatologie. 2. Aufl. Berlin, Heidelberg: Springer; 2010

${ }^{3}$ Dietz HG, Schmittenbecher PP, Slongo T et al., Hrsg. Elastic Stable Intramedullary Nailing (ESIN) in Children. Stuttgart, New York: Thieme; 2006

${ }^{4}$ Schmittenbecher PP, Dietz HG, Linhart WE et al. Complications and problems in intramedullary nailing of children's fractures. Eur J Trauma 2000; 26: 287-293

5 Slongo TF. Complications and failures of the ESIN technique. Injury 2005; 36 (Suppl. 1): A78-A85

6 Vallamshetla VR, De Silva $U$, Bache CE et al. Flexible intramedullary nails for unstable fractures of the tibia in children. An eightyear experience. J Bone Joint Surg $[\mathrm{Br}] 2006$; 88: $536-540$

7 von Laer L, Kraus R, Linhart WE. Frakturen und Luxationen im Wachstumsalter. 5. Aufl. Stuttgart: Thieme; 2007

8 Caterini R, Farsetti P, Ippolito E. Long-term followup of physeal injury to the ankle. Foot Ankle 1991; 11: 372-383

9 Eid AM, Hafez MA. Traumatic injuries of the distal femoral physis. Retrospective study on 151 cases. Injury 2002; 33: 251-255

10 Barmada A, Gaynor T, Mubarak SJ. Premature physeal closure following distal tibia physeal fractures: a new radiographic predictor. J Pediatr Orthop 2003; 23: 733-739

11 Cofield RH, Bryan RS. Acute dislocation of the patella: results of conservative treatment J Trauma 1977; 17: 526-531

12 Sillanpää P, Mattila VM, Iivonen T et al. Incidence and risk factors of acute traumatic primary patellar dislocation. Med Sci Sports Exerc 2008; 40: 606-611

${ }^{13}$ Schöttle P, Beitzel K, Imhoff A. Die kindliche Patellaluxation. Arthroskopie 2009; 22: 51-59

14 Larsen E, Lauridsen F. Conservative treatment of patellar dislocations. Influence of evident factors on the tendency to redislocation and the therapeutic result. Clin Orthop Relat Res 1982: 171: 131-136

15 Vainionpää S, Laasonen E, Silvennoinen T et al. Acute dislocation of the patella. A prospective review of operative treatment. J Bone Joint Surg [Br] 1990; 72: 366-369

16 Salzmann GM, Weber TS, Spang JT et al. Comparison of native axial radiographs with axia MR imaging for determination of the trochlear morphology in patients with trochlear dysplasia. Arch Orthop Trauma Surg 2010; 130: 335-340. Epub 2009 Jun 6
${ }^{17}$ Dejour H, Denjean S, Neyret P. [Treatment of old or recurrent ruptures of the patellar ligament by contralateral autograft]. Rev Chir Orthop Reparatrice Appar Mot 1992; 78: 58-62

18 Schöttle PB, Fucentese SF, Romero J. Clinical and radiological outcome of medial patellofemoral ligament reconstruction with a semitendinosus autograft for patella instability. Knee Surg Sports Traumatol Arthrosc 2005; 13: 516-521

${ }^{19}$ Conlan T, Garth jr. WP, Lemons JE. Evaluation of the medial soft-tissue restraints of the extensor mechanism of the knee. J Bone Joint Surg [Am] 1993; 75: 682-693

${ }^{20}$ Hautamaa PV, Fithian DC, Kaufman KR et al. Medial soft tissue restraints in lateral patellar instability and repair. Clin Orthop Relat Res 1998; 349: 174-182

${ }^{21}$ Nomura E, Inoue $M$. Injured medial patellofemoral ligament in acute patellar dislocation. J Knee Surg 2004; 17: 40-46

22 Nikku R, Nietosvaara Y, Aalto Ket al. Operative treatment of primary patellar dislocation does not improve medium-term outcome: a 7-year follow-up report and risk analysis of 127 randomized patients. Acta Orthop 2005; 76: 699-704

${ }^{23}$ Sillanpää PJ, Mattila VM, Mäenpää $H$ et al. Treatment with and without initial stabilizing surgery for primary traumatic patellar dislocation. A prospective randomized study. J Bone Joint Surg [Am] 2009; 91: 263-273

24 Rudert M. Philipp Edlich P, Wirth CJ. Zügelungsoperation nach Insall bei Luxation oder Subluxation der Patella. Oper Orthop Traumatol 2001; 13: 272-281

${ }^{25}$ Zeichen J, Lobenhoffer P, Bosch U et al. [Interim results of surgical therapy of patellar dislocation by Insall proximal reconstruction]. Unfallchirurg 1998; 101: 446-453

${ }^{26}$ Blauth W, Schwarz I. Operation der habituellen Patellaluxation im Kindesalter. Oper Orthop Traumatol 1989; 1: 94-99

27 Carney JR, Mologne TS, Muldoon M et al. Longterm evaluation of the Roux-Elmslie-Trillat procedure for patellar instability: a 26-year follow-up. Am J Sports Med 2005; 33: 1220 1223

${ }^{28}$ Koskinen SK, Rantanen JP, Nelimarkka OI et al. Effect of Elmslie-Trillat and Roux-Goldthwait procedures on patellofemoral relationships and symptoms in patients with patellar dislocations. Am J Knee Surg 1998; 11: 167-173

${ }^{29}$ Fucentese SF, Schöttle PB, Pfirrmann CW et al. CT changes after trochleoplasty for symptomatic trochlear dysplasia. Knee Surg Sports Traumatol Arthrosc 2007; 15: 168-174

30 Blønd L, Schöttle PB. The arthroscopic deepening trochleoplasty. Knee Surg Sports Traumatol Arthrosc 2010; 18: 480-485
${ }^{31}$ Lephart SM, Pincivero DM, Giraldo JL et al. The role of proprioception in the management and rehabilitation of athletic injuries. Am J Sports Med 1997; 25: 130-137

32 Schottle PB, Romero J, Schmeling A et al. Technical note: anatomical reconstruction of the medial patellofemoral ligament using a free gracilis autograft. Arch Orthop Trauma Surg 2008; 128: 479-484

33 Schöttle PB, Hensler D, Imhoff AB. Die direkte anatomische Rekonstruktion des Lig. patellofemorale mediale in Double-bundle-Technik - „aperture fixation“. Arthroskopie 2008; 21: 192-195

34 Sommerfeldt DW. Die arthroskopisch assistierte Refixation bei knöchernen Ausrissen des vorderen Kreuzbands im Kindes- und Jugendalter. Oper Orthop Traumatol 2008; 20: 310-320

35 Streich AN, Barie A, Gotterbarm T et al. Transphyseal reconstruction of the anterior cruciate ligament in prepubescent athletes. Knee Surg Sports Traumatol Arthrosc 2010; DOI: 10.1007/s00167-010-1057-9

${ }^{36}$ Schneider FJ, Kraus T, Linhart WE. Vorderer Kreuzbandersatz mit der Semitendinosussehne bei Kindern. Oper Orthop Traumatol 2008; 20: 409-422

37 Pećina M, Ivković A, Hudetz D et al. Sagittal osteotomy of the patella after Morscher. Int Orthop 2010; 34: 297-303

${ }^{38}$ Krämer KL, Jani L. Die Operation nach ElmslieTrillat. Oper Orthop Traumatol 1991; 3: 38-48

\section{Dr. med. Dorien Schneidmüller \\ Fachärztin für Orthopädie und Unfallchirurgie}

Dr. med. Emanuel V. Geiger, MBA

Facharzt für Orthopädie

und Unfallchirurgie

Priv.-Doz. Dr. med. Johannes Frank

Leitender Oberarzt

Prof. Dr. med. Ingo Marzi

Ärztlicher Direktor

Klinik für Unfall-, Hand- und

Wiederherstellungschirurgie

Universitätsklinikum

Goethe-Universität Frankfurt

Theodor-Stern-Kai 7

60590 Frankfurt am Main

dorien.schneidmueller@kgu.de 